\title{
To Study the Influence of Resource Allocation on the Average Querying Delay to Reduce the Average Querying Delay
}

\author{
Dr.Shaik Akbar \\ Professor, Dept. of Computer Science \& Engineering \\ Andhra Loyola Institute of Engineering and Technology \\ Vijayawada, A.P., India \\ Sam Abrahmbob \\ M.Tech (CSE) Andhra Loyola Institute of Engineering and Technology \\ Vijayawada, A.P., India
}

\begin{abstract}
By MANETs, refer to both typical MANETs and separated MANETs, otherwise called postpone tolerant systems (DTNs). The previous has moderately thick hub dispersion in a territory while the last has scantily appropriated hubs that meet each other shrewdly. The nearby $P 2 P$ document sharing model gives three points of interest. To begin with, it empowers record contribution when no stand locations are accessible. Next, with the P2P engineering, the block on over-burden servers in present customer server supported record involvement frameworks can be maintained a strategic distance from. Third, it misuses generally squandered shared correspondence opportunities among portable hubs. Thus, hubs can openly and subtly get to and split documents in the appropriated MANET location, which can bolster fascinating applications.
\end{abstract}

Keywords: MANETs, peer-to-peer, file sharing, file availability, P2P

\section{INTRODUCTION}

In particular, current document replication conventions in portable specially appointed systems have two deficiencies. Initially, they do not have a lead to assign restricted assets to various records keeping in mind the end goal to minimize the normal questioning defer. Second, they essentially consider capacity as accessible assets for reproductions; however disregard the way that the record holders' recurrence of meeting different hubs likewise assumes a critical part in deciding document accessibility. Really, a hub that has a higher meeting recurrence with others gives higher accessibility to its records. This turns out to be significantly more obvious in meagerly circulated MANETs, in which hubs meet problematically. In this paper, we present another idea of asset for record replication, which considers both hub stockpiling and meeting recurrence. We hypothetically think about the impact of asset distribution on the normal questioning defer and determine an asset designation control to minimize the normal questioning postpone. We assist propose an appropriated record replication convention to understand the proposed run the show.

\section{RELATED WORK}

Gianuzzi explored the likelihood of procuring a record, which has n copies in the system, from the possibly divided system. He likewise concentrated on the document recovery execution when eradication coding is utilized. Chen talked about how to choose the negligible number of versatile servers expected to guarantee that each information thing can be acquired inside at most $\mathrm{k} \partial \mathrm{k} \_1 \mathrm{p}$ bounces by any hub in the framework. Moussaoui et al. [8] proposed two stages of record replication, essential replication and element replication, to spread copies in the system equitably keeping in mind the end goal to address client issues and anticipate information misfortune on account of system segment.

\section{Problem Definition}

In the previous, repetitive copies are effectively made in the framework, along these lines squandering assets. In the last mentioned, however excess copies are lessened by gathering based participation, 
neighbouring hubs may isolate from each other because of hub portability, prompting expansive question postpone. There are additionally a few works tending to content storing in disengaged MANETs/DTNs for effective information recovery or message steering. They fundamentally reserve information that are every now and again questioned on places that are gone by much of the time by versatile hubs. Both the two classes of replication strategies neglect to completely think about that as a hub's versatility influences the accessibility of its records. Hub versatility, constrained correspondence variety and asset, have make numerous troubles in acknowledging such a P2P record partaking framework. Television can rapidly find records, yet it prompts the telecast storm issue with high vitality utilization. Disregarding endeavours, momentum record replication conventions do not have a run to distribute restricted assets to petitions for copy creation keeping in mind the end goal to accomplish the base normal questioning defer, i.e., worldwide hunt proficiency improvement under constrained assets. They essentially consider capacity as the asset for imitations, however disregard that a hub's recurrence to meet different hubs (meeting capacity in short) additionally impacts the accessibility of its documents. Records in a hub with an advanced convention capacity have advanced accessibility.

\section{Proposed APproach}

In this paper, we present another design of asset for document duplication, which thinks mutually hub stockpiling and hub summit capacity. We hypothetically ponder the impact of asset allotment on the normal questioning defer and infer an ideal document replication run (OFRR) that dispenses assets to every record in light of its prominence and size. We then propose a document replication convention in view of the manage, which approximates the base worldwide questioning defer in a completely dispersed way. We propose a disseminated document replication convention that can roughly understand the ideal record replication administer with the two versatility models in a circulated way. Our trial and re-enactment comes about demonstrate the unrivalled execution of the proposed convention in examination with other delegate replication conventions.

\section{SYSTEM ARCHITECTURE}

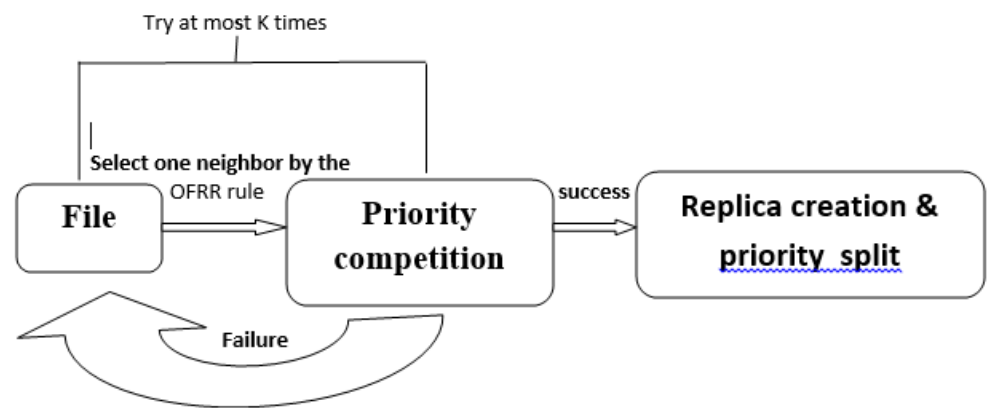

Fig1. Replica System Architecture

As result, the replicas of each file is proportional to their neighbour by replicas nodes when it is success then it replica creation \&priority split done by OFRR. When there is no replicas node connection will be failure.

\section{Proposed methodology}

\subsection{Finest File Reproduction with the RWP Form}

In this we can presume that following exponential distribution is done by congregation time of nodes. The previous encountered node is sovereign with probability of meeting a node. Therefore we define the gathering capability of a node as the average number of nodes it meets in a unit time and use it to investigate the optimal file duplication. Particularly if a node is ability to meet more nodes, By other nodes it having complex which having more chance later on. A node's possibility of being encounter by other nodes is relative to the meeting ability of the node. This specifies that files reside in nodes with higher meeting ability have higher availability than files in nodes with lower meeting capability. The measure a node's resource in the report of both meeting ability and storage. When the node occupies memory there is a possible of create replica on node. Also, its probability of being met by others is unfaltering by the node's meeting ability. The storage resource and the meeting ability resource of the both on node that called replica naturally guzzle. 

Querying Delay

\subsection{Area Based Mobility Form}

In this Area-based mobility form the analysis is done. Each node's fulfilling ability should be considered. It is defined as a node's ability to satisfy queries in the system and is calculated based on the node's facility to satisfy queries in each community. In this model, since nodes' file comfort is established during a certain time period, we measured (i.e., enquiring rates for altered files) period of time each node's file querying blueprint remains stable. When a file generate in a community by the amount of queries represent by the number of nodes in an area. As an outcome, a tiny area satisfies queries of small ability file holder. The adding up of the satisfying ability is done integrates with every community's tiny proportion node.

\subsection{Summit Capacity Allocation}

In this model meeting ability division measured from real traces we consider node meeting ability as an important factor of our instruction in the resource allocation. For every outline, we ranked them in declining order by the meeting ability of nodes measured. By see that in every outlines, wide range spread done by node meeting ability. The different meeting abilities of usually nodes that match with our previous assert. Also, it verify the node meeting ability as a resource in file duplication is necessary since if all meeting ability of nodes are comparable, replicas on different nodes have similar probability to meet requesters, and hence meeting ability in resource allocation not to be judge.

\subsection{Design of the File Replication Protocol}

We suggest the precedence rivalry and split file replication protocol (PCS). We first say how a node retrieves the parameters needed and then observe the detail of PCS. In PCS, each node meeting ability informs energetically and the average summit facility of all nodes in the structure. Such neighbour nodes swap over information. The process of the replication of a file establish in PCS. Stand on OFRR, since an organizer (File) with a higher P should receive more resources; a node should assign higher main concern to its files with higher $\mathrm{P}$ to participate resource with other nodes. Thus, every node instructs all of its files in sliding order of their Ps and forms models for the files in a top-down approach at regular intervals. The organizer (File) replication stops when the statement session of the two occupied nodes ends. Then, every node continues the duplication developments for its files after away from each other from the detached node from the neighbour node list. Since file popularity, Ps, and accessible system resources change as time goes on, every node periodically executes PCS to vigorously handle these time-shifting factors. Each node also episodically calculates the reputation of its files (qj) to replicate the changes on file popularity (due to node querying pattern and rate changes) in different time periods. The review file reputation keep informed can repeatedly handle file zip.

\section{RESULTS}

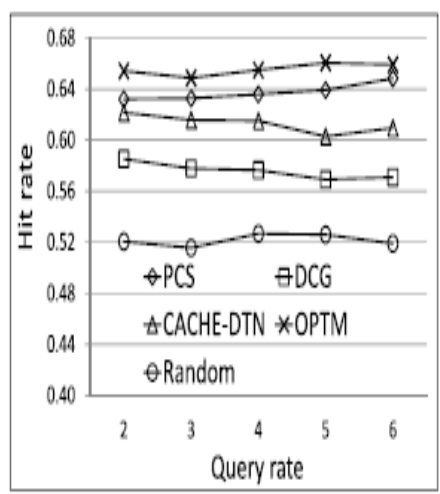

(a) Hit rate.

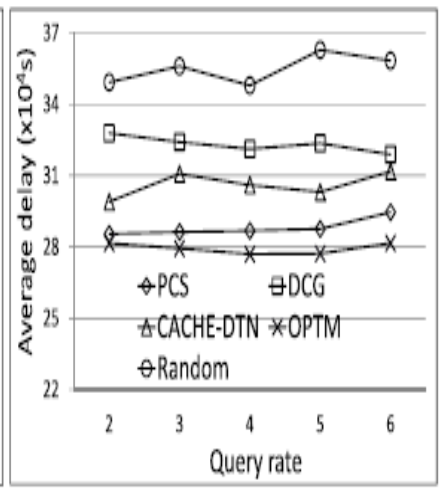

(b) Average delay.

Fig2. Show of file duplication protocol with the barter Sketch

Demonstrate the CDF of the extent of assets allotted to reproductions in every convention in the experiments with the Wrangle follow and the MIT actuality follow, individually. We observe in mutually figures, PCS exhibits secure closeness to OPTM and the previous two take after CACHEDTN_PCS. Arbitrary likewise introduces close similitude to OPTM on the CDF bend. In any case, the 
contrast in the middle of PCS and casual is that PCS relegates need for mainstream documents while arbitrary provides still need to every record. While mainstream documents are questioned the entire extra as often as possible, Random still prompts a low execution. For last three techniques that support prevalent records, we discover that the nearer closeness with OPTM a convention has the improved general execution it has. Such an outcome likewise coordinates with what we saw in the analysis in associated MANETs. This demonstrates the accuracy of our hypothetical examination and the ensuing OFRR manage for separated MANETs.

\section{Conclusion}

We first hypothetically examined the impact of imitation dissemination on the normal questioning postpone under obliged accessible assets with two portability models, and afterward inferred an ideal replication decide that can assign assets to document copies with insignificant normal questioning defer. At last, we composed the need rivalry and rip duplication gathering (PCS) that understands the ideal duplication manage in a completely conveyed way. We examined the issue of how to apportion restricted assets for document replication with the end goal of worldwide ideal record seeking productivity in MANETs.

\section{REFERENCES}

[1] “Qik," http://qik.com/, 2014.

[2] "Flixwagon," http://www.flixwagon.com/, 2014.

[3] C. Palazzi and A. Bujari, "A Delay/Disruption Tolerant Solution for Mobile to Mobile File Sharing," Proc. IFIP/IEEE Wireless Days, 2010.

[4] Y. Tseng, S. Ni, and E. Shih, "Adaptive Approaches to Relieving Broadcast Storms in a Wireless Multihop Mobile Ad HocNetwork," Proc. 21st Int'l Conf. Distributed Computing Systems(ICDCS), pp. 481-488, 2001.

[5] B. Chiara et al., "HI Bop: A History Based Routing Protocol for Opportunistic Networks," Proc. IEEE Int'l Symp. World of Wireless, Mobile and Multimedia Networks (WoWMoM), 2007.

[6] A. Lindgren, A. Doria, and O. Schelen, "Probabilistic Routing in Intermittently Connected Networks," ACM SIGMOBILE Mobile Computing and Comm. Rev., vol. 7, no. 3, pp. 19-20, 2003.

[7] F. Li and J. Wu, "MOPS: Providing Content-Based Service in Disruption-Tolerant Networks," Proc. IEEE 29th Int'1 Conf. Distributed Computing Systems (ICDCS), 2009.

[8] S. Moussaoui, M. Guerroumi, and N. Badache, "Data Replicationin Mobile Ad Hoc Networks," Proc. Second Int'l Conf. Mobile Ad-hoc and Sensor Networks (MSN), pp. 685-697, 2006.

[9] L. Yin and G. Cao, "Supporting Cooperative Caching in Ad HocNetworks," IEEE Trans. Mobile Computing, vol. 5, no. 1, pp. 77-89, Jan. 2006.

[10] T. Hara and S.K. Madria, "Data Replication for Improving Data Accessibility in Ad Hoc Networks," IEEE Trans. Mobile Computing, vol. 5, no. 11, pp. 1515-1532, Nov. 2006.

[11] J. Zheng, J. Su, K. Yang, and Y. Wang, "Stable Neighbour Based Adaptive Replica Allocation in Mobile Ad Hoc Networks," Proc.Int'1 Conf. Computational Science (ICCS), 2004.

[12] H. Duong and I. Demure, "Proactive Data Replication Semantic Information within Mobility Groups in MANET," Proc. SecondInt'l Conf. Mobile Wireless Middleware, Operating Systems, and Applications (Mobil ware), 2009.

[13] Y. Huang et al., "Optimizing File Retrieval in Delay-Tolerant Content Distribution Community," Proc. Int'l Conf. Distributed Computing Systems (ICDCS), 2009.

[14] W. GAO, G. Cao, A. Iyengar, and M. Srivatsa, "Supporting Cooperative Caching in Disruption Tolerant Networks,” Proc. Int'l Conf. Distributed Computing Systems (ICDCS), 2011.

[15] J. Reich and A. Chaintreau, "The Age of Impatience: Optimal Replication Schemes for Opportunistic Networks," Proc. Fifth Int'lConf. Emerging Networking Experiments and Technologies (Context), 2009. 


\section{AUTHORS' BIOGRAPHY}

Dr.Shaik Akbar, received M.Sc (Computer Science) from Acharya Nagarjuna University, M.Tech (CS\&T) from Andhra University and Ph.D from GITAM University. Presently working as a Professor in Andhra Loyola Institute of Engineering and Technology, Vijayawada, Affiliated to J.N.T.U, Kakinada. My area of interest is Intrusion Detection, Network Security, Colud Computing, Bigdata and Algorithms

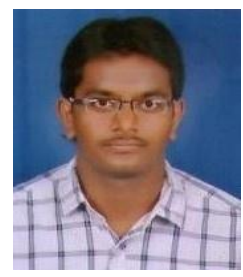

Sam Abrahmbob, B.Tech from JNTU, Kakinada and M.Tech (CSE) from Andhra Loyola Institute of Engineering and Technology, Vijayawada, A.P., India. My area of interest is Network Security and Algorithms. 